\title{
A Parametric Study on the Innovative Coupling Arm Connecting the Coupled TAD-TLP
}

\author{
Xiangbo Liu ${ }^{1,2}$, Allan Ross Magee ${ }^{1,2, *}$, Aziz Merchant ${ }^{1,3}$, Anis Hussain $^{1,3}$, \\ Ankit Choudhary ${ }^{1,3}$, Amit Jain ${ }^{1,3}$, and Bernad A.P Francis ${ }^{1,3}$ \\ ${ }^{1}$ Keppel-NUS Corporate Laboratory, Singapore \\ ${ }^{2}$ Department of Civil and Environmental Engineering, National University of Singapore, Singapore \\ ${ }^{3}$ Keppel Offshore and Marine Technology Centre, Singapore
}

\begin{abstract}
Two body marine systems, like a Tender Assisted Drilling (TAD) vessel coupled with a floating Dry-Tree Unit (DTU), have become very common in offshore operations. One of the unavoidable challenge we have to cope with is the connection between the TAD and DTU should make sure the TAD does not drift away from the platform and also avoid the possible collision in case of a harsher environment. The objective of this study is to understand the hydrodynamic interactions between the two coupled floating bodies and improve the devising of the innovative connection system. In this study, an innovative rigid connection system, the coupling arm is applied to connect the TAD and a DTU, in this case, a Tension Leg Platform (TLP). The whole system is modelled by the commercial software HARP. A comprehensive parametric study on the pretension and the nominal length of the coupling arm is carried out. The hydrodynamic analysis of the coupled TAD-TLP system elucidates the interactions between the two bodies. The chosen combination of the coupling arm pretension and the nominal length will determine the required stroke range and maximum forces needed to design the innovative coupling arm for safe operations.
\end{abstract}

\section{Introduction}

A TAD vessel operates an erectable drilling rig deployed on another nearby platform, called a wellhead platform (WHP) or a floating DTU. The TAD hosts much of the heavy drilling equipment, like pipe, mud and drilling quarters and is stationed alongside the DTU from which the rig will operate. This load sharing reduces the loads on the DTU. While operating, the TAD needs to be structurally coupled with the drilling platform. The coupling makes sure that the TAD does not drift away from the platform and limits the relative motions between the TAD and the DTU. This kind of two-body marine system recently becomes very common in offshore operations, as shown in Fig.1. The coupling between floating structures varies depending on the nature of the job to be performed.

\footnotetext{
*Corresponding author: ceemar@nus.edu.sg
} 


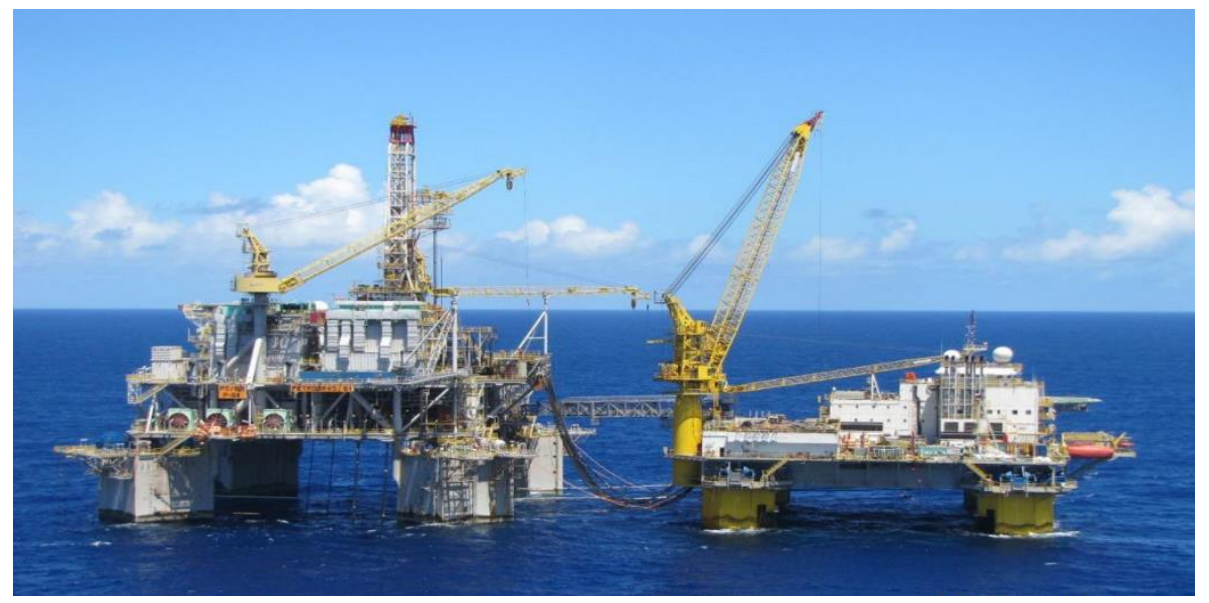

Fig. 1. Side by side floating bodies at sea

Several researches have been carried out on the prediction of motion responses and hydrodynamic interactions of side-by-side floating structures in waves. Inoue and Islam (1999) predict the relative motion between LNG-FPSO and LNG carrier by using a threedimensional source link method in frequency and time domain. Chen $(2001,2002)$ used three-dimensional source distribution method to predict the wave exciting forces, relative motion, wave elevation and drift force between two bodies in waves. Buchner et al. (2001) developed numerical simulation model for hydrodynamic response of LNG-FPSO with alongside moored LNG carrier in time domain. Choi and Hong (2002) used the higherorder boundary element method to analyse the hydrodynamic interaction between multiple floating bodies in waves.

However, the above-mentioned studies do not consider the interconnection between the two floating bodies. The connection between the tender and the platform can be considered as a constraint that can restrict the motion of the tender. If the connection is rigid enough, the tender and the platform will move together with the same motion. However, in the most situations, the "soft" connection is widely adopted to provide a certain degree of freedom. Therefore, the independent motion between the tender and the platform will occur, which may further lead to the possible collision if the motion is large. In case of a harsher environment, these two bodies need to be separated. The present practice of connecting two bodies by hawsers ropes creates several problems in such situation. Limited literature on the interconnection between two floating structures could be found. Xia and Taghipour (2012) investigated the feasibility of a TLP with TAD using a semisubmersible tender vessel for filed developments offshore North West Australia. They still applied the hawsers as interconnections between TLP and TAD. Moreover, the weather window for the safe operation under such circumstances is also an unclear question.

Therefore, in this study, an innovative rigid connection system, the coupling arm, is applied to connect the TAD and TLP. The hydrodynamic analysis reported here is carried out by using the commercial software HARP. In order to shed further light on understanding of hydrodynamic interactions of coupled TAD and TLP system, a comprehensive parametric study on the pretension and nominal length of the coupling arm is performed. The chosen combination from this parametric study will further improve the devising of this kind of innovative connection system. 


\section{Theoretical background}

The prediction of wave forces and moments on structures is one of the primary tasks in the simulation of the body motion. In this study, the Cummins method (Pinkster and Van Oortmerssen 1977) is used to evaluate the wave force $\mathbf{F}^{p}$ and moment $\mathbf{M}^{p}$ applied on the TAD and the TLP operating nearby, in which the subscripts $p=1,2$ represent the quantities for the TAD and TLP respectively. In all the subsequent study, the hydrodynamic moment $\mathbf{M}^{p}$ is further defined as

$$
M_{k-3}^{P}(t)=F_{k}(t), \quad(p=1,2 ; k=4,5,6) .
$$

The generalized hydrodynamic force can be written as a sum of several components:

$$
\mathbf{F}^{p}(t)=\mathbf{F}_{S}^{p}(t)+\mathbf{F}_{R}^{p}(t)+\mathbf{F}_{E}^{p}(t), \quad(p=1,2),
$$

where $\mathbf{F}_{S}^{p}, \mathbf{F}_{R}^{p}$ and $\mathbf{F}_{E}^{p}$ represent the hydrostatic restoring force, radiation force and wave exciting force respectively.

The hydrostatic restoring force is induced by the hydrostatic pressure change due to the motion of the body. For the body with a vertical wall at the free surface and finite motion amplitude, $\mathbf{F}_{S}^{p}$ is conventionally assumed to be linear with the motion response of the body, $\boldsymbol{\Xi}^{p}$. After obtaining the hydrostatic restoring stiffness matrix [C] for the two-body system, $\mathbf{F}_{S}^{p}$ can then be approximately calculated.

In the application of the Cummins method, an arbitrary body motion is described as a succession of small impulsive displacements. The total fluid reactive force is the sum of the reactions to the individual impulsive displacements, each reaction being calculated with an appropriate time lag from the instant of the corresponding impulsive motion. Then components of the radiation force vector, $\mathbf{F}_{R}^{p}$, can be computed by the following equation in the time-domain:

$$
\begin{gathered}
F_{R, k}^{p}(t)=-\sum_{j=1}^{6}\left[a_{l j}(\infty) \ddot{\Xi}_{j}^{1}(t)+\int_{-\infty}^{t} K_{l j}(t-\tau) \dot{\Xi}_{j}^{1}(\tau) d \tau\right] \\
-\sum_{j=7}^{12}\left[a_{l j}(\infty) \ddot{\Xi}_{j-6}^{2}(t)+\int_{-\infty}^{t} K_{l j}(t-\tau) \dot{\Xi}_{j-6}^{2}(\tau) d \tau\right], \quad(l=k+6 p-6 ; k=1 \ldots, 6 ; p=1,2),
\end{gathered}
$$

where $a_{l j}(\infty)$ is a constant equivalent to the added mass of the two-body system at infinite frequency, the convolution integral represents the memory effects of the wave forces on the body from the wave radiation generated by body motion prior to the time $t$, and $K_{l j}$ is called the retardation function which is related to the frequency-domain solution of the radiation problem as follow:

$$
K_{l j}(t)=\frac{2}{\pi} \int_{0}^{\infty} b_{l j}(\omega) \cos \omega t d \omega, \quad(l, j=1, \ldots, 12),
$$

where $b_{l j}$ is the frequency domain radiation damping coefficient coupling modes $l$ and $j$. For the system consisting of 2 bodies, the added mass and radiation damping of one body 
can be induced by not only the motion of itself but also that of the nearby body. To obtain the $a_{l j}(\infty)$ and $b_{l j}$, we have to solve the $6 \times 2$ radiation problems.

Waves in the real environment are also random or irregular. For an irregular unidirectional wave process, the wave elevation can be represented by summation of a large number of the individual wave components. Accurate to the second-order wave steepness, the irregular wave profile at the reference point can be written as:

$$
\begin{gathered}
\zeta(t, \mathbf{x})=\zeta^{(1)}(t, \mathbf{x})+\zeta^{(2)}(t, \mathbf{x})+O\left(\varepsilon^{3}\right) \\
=\sum_{j=1}^{N} A_{j} \cos \left(\kappa_{j} x-\omega_{j} t+\beta_{j}\right)+\sum_{j=1}^{N} \sum_{l=1}^{N}\left[A_{j l}^{+} \cos \left(\kappa_{j l}^{+} x-\omega_{j l}^{+} t+\beta_{j l}^{+}\right)+A_{j l}^{-} \cos \left(\kappa_{j l}^{-} x-\omega_{j l}^{-} t+\beta_{j l}^{-}\right)\right]+O\left(\varepsilon^{3}\right),
\end{gathered}
$$

in which, superscripts (1) and (2) represent the first-order and second-order quantities; $\varepsilon$ is the wave steepness; $N$ is the number of linear wave components; $A_{j}, \omega_{j}$ and $\beta_{j}$ are the wave amplitude, angular frequency and the initial phase of the $j$ th wave component; $\omega_{j l}^{ \pm}=\omega_{j} \pm \omega_{l} ; \kappa_{j l}^{ \pm}=\kappa_{j} \pm \kappa_{l} ; \beta_{j l}^{ \pm}=\beta_{j} \pm \beta_{l} ; A_{j l}^{+}$and $A_{j l}^{-}$represent the amplitude of the sum- and difference-frequency components, and are proportional to $A_{j} A_{l}$ and $A_{j} A_{l}^{*}$ respectively.

The first-order exciting forces on bodies induced by the incident and the diffraction waves can be computed by a Volterra series model as follows in the time-domain

$$
F_{E, k}^{p(1)}(t)=\int_{-\infty}^{\infty} h_{k}^{p}(t-\tau) \zeta^{(1)}(\tau) d \tau, \quad(k=1, \ldots, 6 ; p=1,2),
$$

where $h_{k}^{p}(t)$ is the linear impulse response function, which represents the exciting force at time $t$ due to a unit amplitude wave input at $\tau$ :

$$
h_{k}^{p}(t-\tau)=\operatorname{Re}\left[\frac{1}{\pi} \int_{0}^{\infty} H_{k}^{p}(\omega) e^{i \omega(t-\tau)} d \omega\right], \quad(k=1, \ldots, 6),
$$

where ' $\mathrm{Re}$ ' indicates that the real part is to be taken; $H_{k}^{p}$ is called the linear transfer function (LTF), which represents the linear exciting forces from a unit amplitude regular incident wave at frequency $\omega . H_{k}^{p}$ defines the relation between the wave elevation and the first-order exciting forces in mode $k$.

The second-order time-domain exciting forces contain the sum-frequency and the difference-frequency parts:

$$
F_{E, k}^{p(2)}(t)=F_{E, k}^{p(2)+}(t)+F_{E, k}^{p(2)-}(t), \quad(k=1, \ldots, 6 ; p=1,2) .
$$

When using the Cummins method, the calculation of the second-order time-domain exciting forces applied on a body are conventionally computed based on the QTFs via a two-term Volterra series model. Then the force in mode $k$ is represented as 


$$
F_{E, k}^{p(2) \pm}(t)=\int_{-\infty}^{+\infty} \int_{-\infty}^{\infty} h_{k}^{p \pm}\left(t-\tau_{j}, t-\tau_{l}\right) \zeta^{(1)}\left(\tau_{j}\right) \zeta^{(1)}\left(\tau_{l}\right) d \tau_{j} d \tau_{l}, \quad(k=1, \ldots, 6 ; p=1,2)
$$

where $h_{k}^{p \pm}$ represents the exciting forces at the time $t$ due to two unit amplitude incident waves at the times $\tau_{j}$ and $\tau_{l}$, and can be obtained by a Fourier transform of quadratic transfer function (QTF) $H_{j l, k}^{p \pm}$ :

$$
h_{k}^{p \pm}\left(t-\tau_{j}, t-\tau_{l}\right)=\operatorname{Re}\left[\frac{1}{2 \pi^{2}} \int_{0}^{\infty} \int_{0}^{\infty} H_{j l, k}^{p \pm} e^{i\left[\omega_{j}\left(t-\tau_{j}\right) \pm \omega_{j}\left(t-\tau_{l}\right)\right]} d \omega_{j} d \omega_{l}\right], \quad(k=1, \ldots, 6 ; p=1,2),
$$

where, $H_{j l, k}^{p \pm}$ represents the second-order exciting forces in mode $k$ on a body due to bichromatic incident waves with unit amplitudes.

As illustrated in Eqs. (3), (6) and (9), the wave force and moment time series in the timedomain can be generated by utilizing the hydrodynamic coefficients, such as added mass, radiation damping, LTF and QTF. These unknown coefficients can be determined through a frequency-domain analysis. The HYDRO module of the commercial software HARP is developed based on the second-order wave diffraction theory explained above. In this study, HARP is used to perform the multi-body hydrodynamic analysis and generate the frequency-domain solution.

\section{Numerical modelling}

In this study, a generic TAD and a generic TLP are modelled to perform the numerical simulations in HARP. Table 1 provides the key parameters of the TAD and TLP. The water depth of the field is $1180 \mathrm{~m}$.

Table 1. Key parameters of the TAD and TLP.

\begin{tabular}{|ccc|}
\hline Designation & Unit & Value \\
\hline & TAD key particulars & \\
\hline Displacement & Mt & 21018 \\
Column length & $\mathrm{m}$ & 13.1 \\
Column breadth & $\mathrm{m}$ & 9.1 \\
Pontoon height & $\mathrm{m}$ & 6.1 \\
Pontoon length & $\mathrm{m}$ & 93.9 \\
Pontoon breadth & $\mathrm{m}$ & 12.5 \\
Distance between pontoons & $\mathrm{m}$ & 34.5 \\
Draft & $\mathrm{m}$ & 15.2 \\
\hline & TLP key particulars & \\
\hline Displacement & $\mathrm{Mt}$ & 40636 \\
Column size (4) $(\mathrm{L} \times \mathrm{W} \times \mathrm{Ht})$ & $\mathrm{m} \times \mathrm{m} \times \mathrm{m}$ & $15 \times 15 \times 45$ \\
Column spacing $(\mathrm{c} / \mathrm{c})$ & $\mathrm{m}$ & 55 \\
Pontoon height & $\mathrm{m}$ & 8 \\
Pontoon length & $\mathrm{m}$ & 19.8 \\
Pontoon breadth & $\mathrm{m}$ & 7 \\
Draft & $\mathrm{m}$ & 29 \\
\hline
\end{tabular}


According to the particulars provided in Table 1, the hydrodynamic models are established. Fig.2 illustrates the generated side by side panel model.

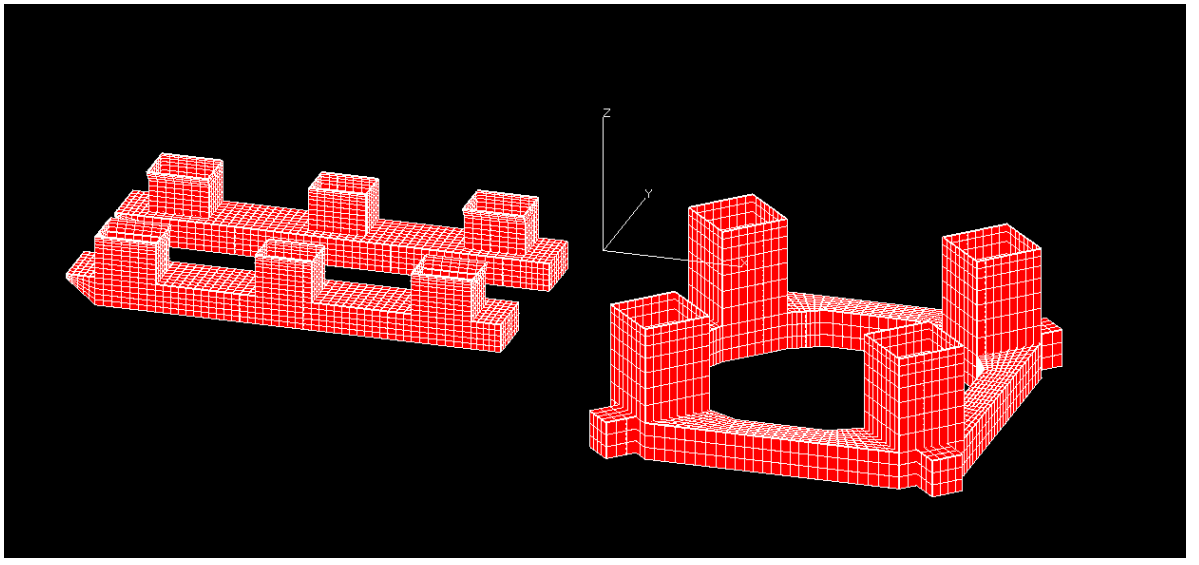

Fig. 2. Underwater panel models for numerical simulation.

In this study, to model the rigid innovative connection system between the TAD and TLP, the piston-type coupling arm (Jain et al., 2016) is applied. Fig.3 provides the sketch of the coupled TAD and TLP connected by the coupling arm. As shown in Figure 3, two coupling arms are applied in all the numerical simulations. According to Yang et al. (2007), the response of the coupling arm is governed by,

$$
F=F_{0} /\left(1-\frac{Z}{Z_{0}}\right)^{C}
$$

where $F_{0}$ is the pretension of the coupling arm, $Z_{0}$ denotes the nominal length at calm water position, $\mathrm{c}$ is the gas constant equals 1.1 in this study, $\mathrm{F}$ and $\mathrm{Z}$ are the force from the coupling arm and its relative motion respectively. Thus, the stiffness is $F_{0} * c / Z_{0}$ at calm water position. In the commercial software HARP, a user-defined subroutine is created as an external source file to simulate the coupling arm.

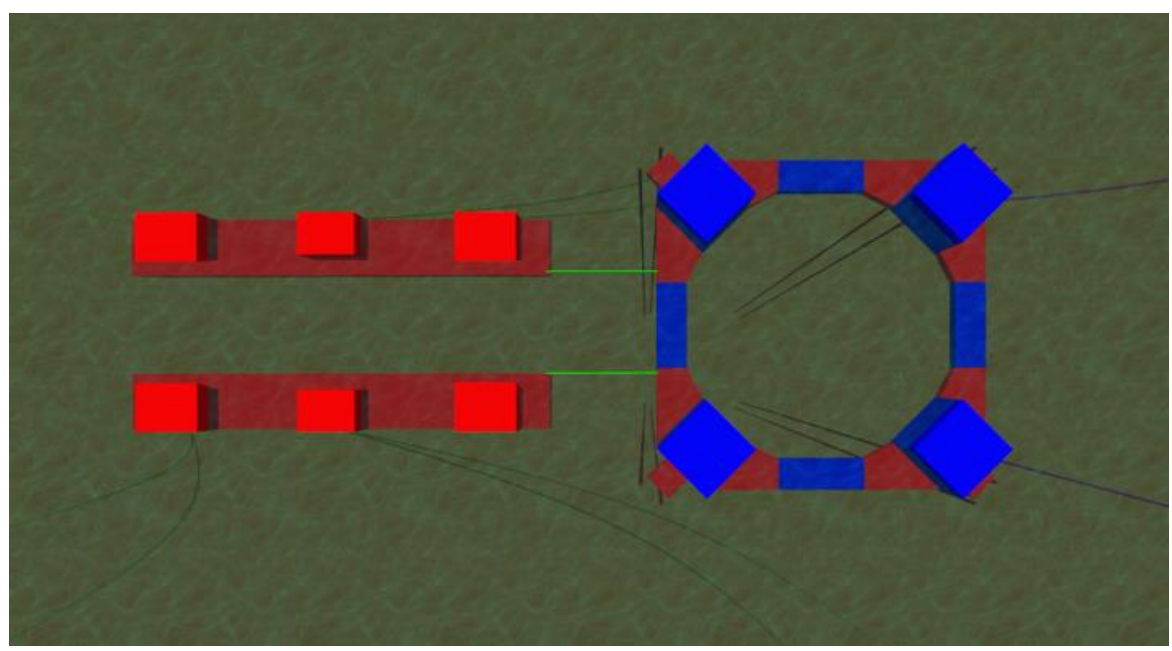

Fig. 3. A sketch of the coupled TAD and TLP connected by two coupling arms. 
In this study, the 1-year max wave condition for the Southeast Brazilian coast is applied to all the numerical simulations. The corresponding metocean data is summarized in Table 2. It should be noted that the wave, wind and current are assumed to be collinear for the hydrodynamic analysis.

Table 2. Metocean data- 1-year max wave.

\begin{tabular}{|c|c|c|}
\hline Environment & Unit & Quantity \\
\hline \multicolumn{3}{|l|}{ Wave (Jonswap) } \\
\hline Significant wave height & $\mathrm{m}$ & 6.2 \\
\hline Peak spectrum period & secs & 12.5 \\
\hline Max wave height & $\mathrm{m}$ & 11.6 \\
\hline Gamma & & 1.9 \\
\hline \multicolumn{3}{|l|}{ Wind (NPD) } \\
\hline 1-hour@10 m elevation & $\mathrm{m} / \mathrm{s}$ & 15.9 \\
\hline Direction & degree & 0 \\
\hline \multirow[t]{13}{*}{ Current velocity profile } & Depth (m) & Velocity $(\mathrm{m} / \mathrm{s})$ \\
\hline & 0 & 0.96 \\
\hline & -50 & 0.96 \\
\hline & -100 & 0.81 \\
\hline & -250 & 0.72 \\
\hline & -350 & 0.52 \\
\hline & -450 & 0.48 \\
\hline & -550 & 0.38 \\
\hline & -650 & 0.30 \\
\hline & -750 & 0.23 \\
\hline & -950 & 0.23 \\
\hline & -1050 & 0.23 \\
\hline & -1180 & 0.08 \\
\hline
\end{tabular}

\section{Results and discussion}

The main objective of this study is to investigate the hydrodynamic performance of the TAD-TLP system connected by the coupling arm. According to Eq. (11), the pretension and the nominal length of the coupling arm at calm water position are the key parameters that affect the mechanism of the coupling arm. Therefore, a series of numerical simulations are carried out regarding the pretension and the nominal length of the coupling arm.

To begin with, the pretension is chosen to $500 \mathrm{kN}$ for each coupling arm, while the nominal length varies from $5 \mathrm{~m}$ to $9 \mathrm{~m}$. Apply the user-defined subroutine into HARP, the following results are obtained. Fig. 4 shows the time history of the relative motion of the coupling arm. It should be noted that the results are from only one coupling arm. In this study, the direction of wave, wind and current is 0 degree. Therefore, identical results are achieved for both coupling arms. In addition, since results of $Z_{0}=8 \mathrm{~m}$ are quite close to those of 7 and $9 \mathrm{~m}$, they are not displayed here in Fig.4. It can be seen that similar results are obtained for $6-9 \mathrm{~m}$. Larger motions can be observed for $\mathrm{Z}_{0}=5 \mathrm{~m}$. To further analyse the performance, the spectrum of the relative motions at range of low frequency and wave frequency are shown in Fig.5. Identical results at wave frequency are achieved. It suggests that under pretension of $500 \mathrm{kN}$, different nominal lengths applied here won't affect the 
response at wave frequency. The outstanding results at left side suggest that the difference is mainly due to response at low frequency.

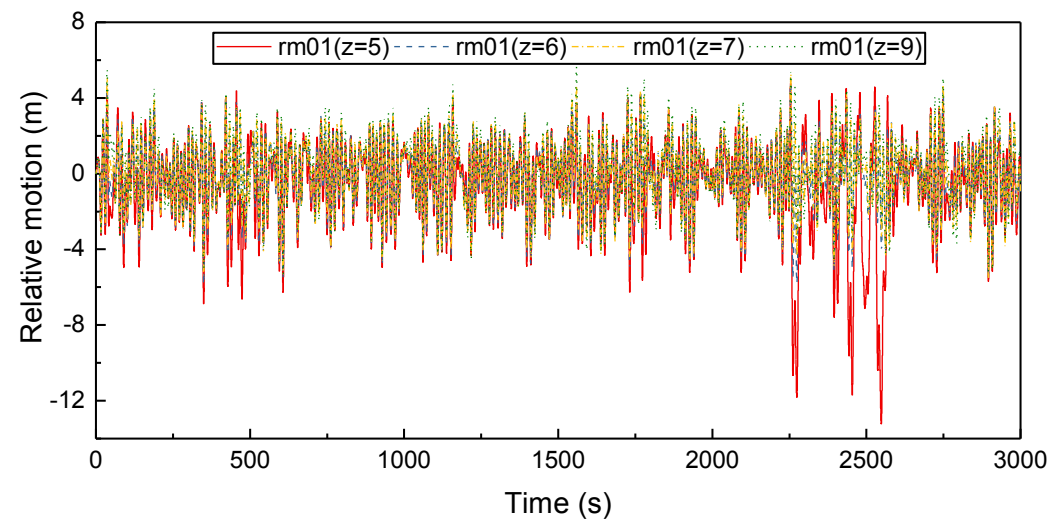

Fig. 4. Time history of the relative motions of different nominal lengths under pretension $500 \mathrm{kN}$.
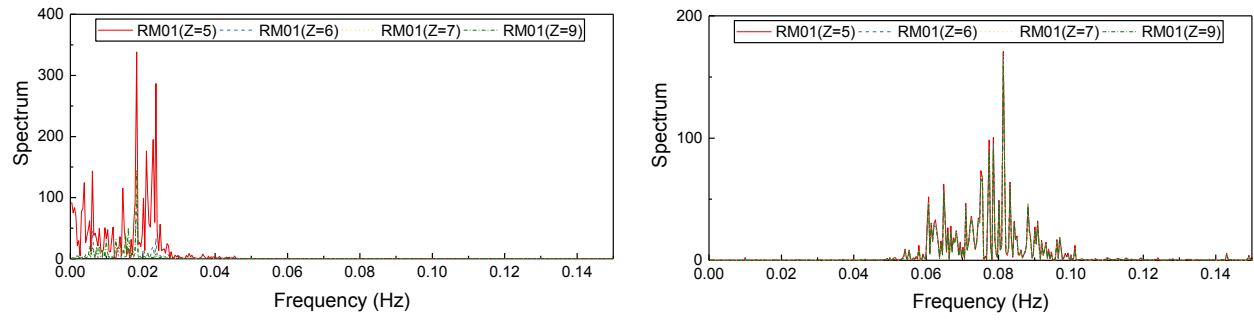

Fig. 5. Spectrum of the relative motions (left : low frequency, right : wave frequency).
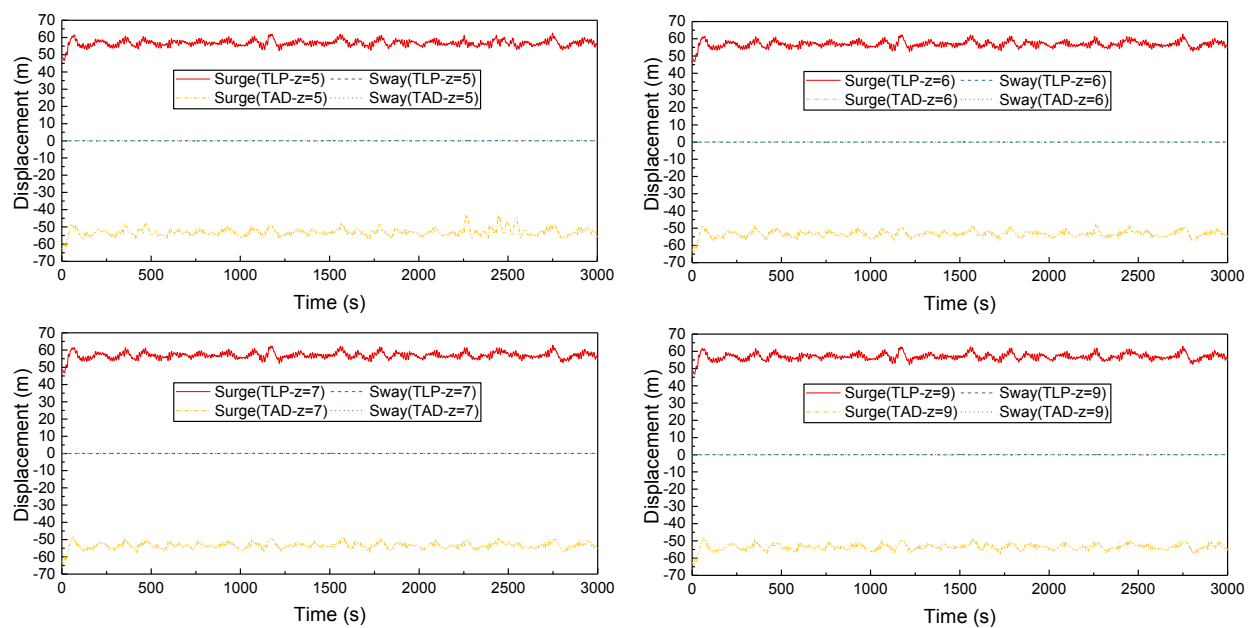

Fig. 6. Time history of body motions (left upper: $Z_{0}=5$, right upper: $Z_{0}=6$, left lower: $Z_{0}=7$, right lower: $\left.\mathrm{Z}_{0}=8\right)$.

Fig. 6 provides the time history of the body displacement of TAD and TLP. There are almost no sway motions of both bodies under the 0 degree of wave, wind and current direction. Therefore, surge motions are further compared for different nominal lengths of coupling arm, as shown in Fig.7 and 8. Consistent with the relative motions of the coupling arm, larger body motions are observed for $Z_{0}=5 \mathrm{~m}$, especially for the TAD. Responses at 
wave frequency are quite close to each other while at low frequency, differences can be observed.
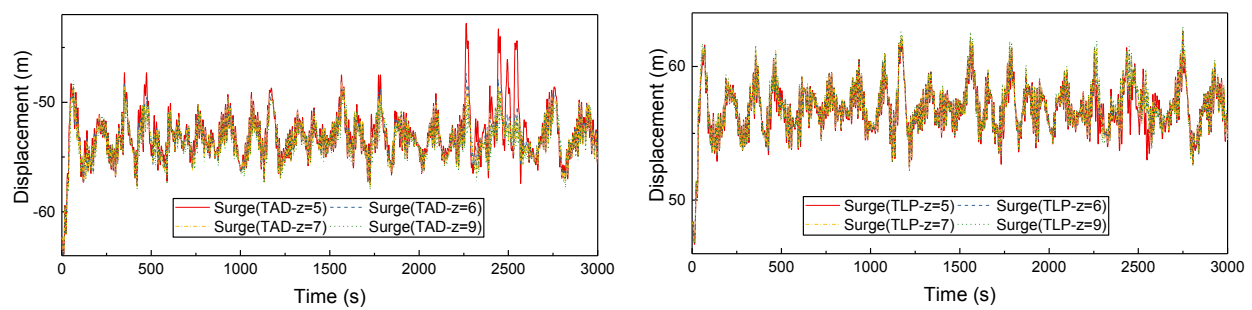

Fig. 7. Time history of surge motions (left : TAD, right : TLP).
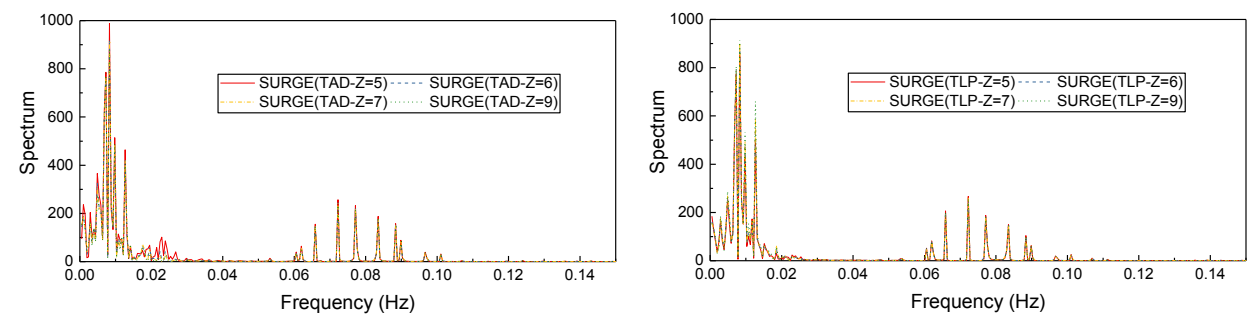

Fig. 8. Spectrum of surge motions (left : TAD, right : TLP).

To better reveal the performance of the coupling arm, the stats for relative motions at low frequency and wave frequency are obtained, displayed in Fig. 9 and provided in Table 3 . Corresponding to the previous observation, relative motions at wave frequency are quite close to each other. Except $\mathrm{Z}_{0}=5 \mathrm{~m}$, relative motions of the coupling arm under pretension $500 \mathrm{kN}$ dominate at wave frequency. The estimations of the stroke range (trough to peak) of the coupling arm are also made based on the Weibull distribution. From the results shown in Fig.10, it can be concluded that $Z_{0}=7,8$ and $9 \mathrm{~m}$ would achieve more promising results than the rest of the two. Therefore, these three values are chosen to carry out further analysis.
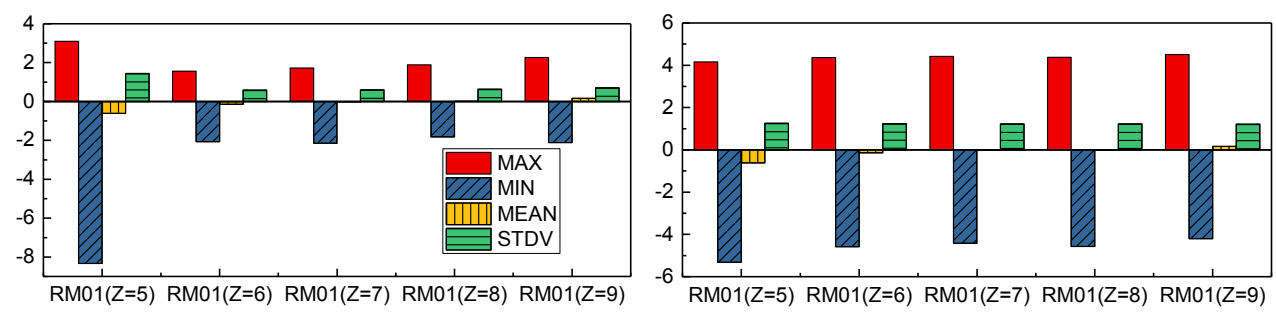

Fig. 9. Stats of relatvie motions of coupling arm (left : low frequency, right : wave frequency). 
Table 3. Stats of relative motions at low and wave frequncy.

\begin{tabular}{ccccc}
\hline & MAX & MIN & MEAN & STDV \\
\hline Low frequency & $(\mathrm{m})$ & & & \\
RM01 $\left(Z_{0}=5\right)$ & 3.097 & -8.331 & -0.616 & 1.437 \\
RM01 $\left(Z_{0}=6\right)$ & 1.555 & -2.068 & -0.14 & 0.581 \\
RM01 $\left(Z_{0}=7\right)$ & 1.718 & -2.144 & -0.009 & 0.6 \\
RM01 $\left(Z_{0}=8\right)$ & 1.884 & -1.819 & 0.008 & 0.635 \\
RM01 $\left(Z_{0}=9\right)$ & 2.267 & -2.109 & 0.165 & 0.704 \\
\hline Wave frequency & $(m)$ & & & \\
RM01 $\left(Z_{0}=5\right)$ & 4.156 & -5.317 & -0.616 & 1.258 \\
RM01 $\left(Z_{0}=6\right)$ & 4.365 & -4.586 & -0.14 & 1.238 \\
RM01 $\left(Z_{0}=7\right)$ & 4.415 & -4.422 & -0.009 & 1.229 \\
RM01 $\left(Z_{0}=8\right)$ & 4.372 & -4.572 & 0.008 & 1.221 \\
RM01 $\left(Z_{0}=9\right)$ & 4.506 & -4.202 & 0.165 & 1.217 \\
\hline
\end{tabular}

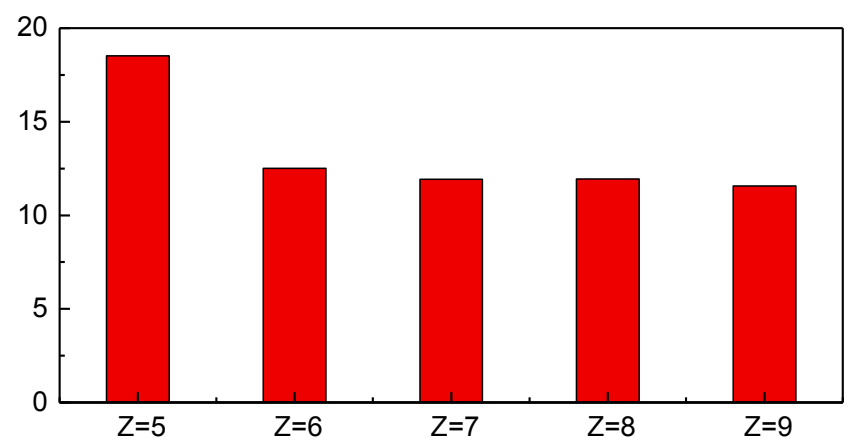

Fig. 10. Estimation of the stroke range of the coupling arm.

Similarly, eight different pretension from $1000 \mathrm{kN}$ to $8000 \mathrm{kN}$ are chosen to perform the numerical simulations while the nominal lengths are $Z_{0}=7,8$ and $9 \mathrm{~m}$. Time histories and spectrums of the relative motions and displacement of the TAD and TLP are obtained just like those in the previous analysis. To directly investigate the performance of the coupling arm, the stats of relative motions at low frequency and wave frequency for different pretension are displayed in Figs.11-13. Consistent with the previous observations, wave frequency dominates for most cases. However, different from the previous one, relative motions at wave frequency are obviously not close to each other. This might be due to the relatively larger difference in stiffness caused by different pretension of the coupling arm.
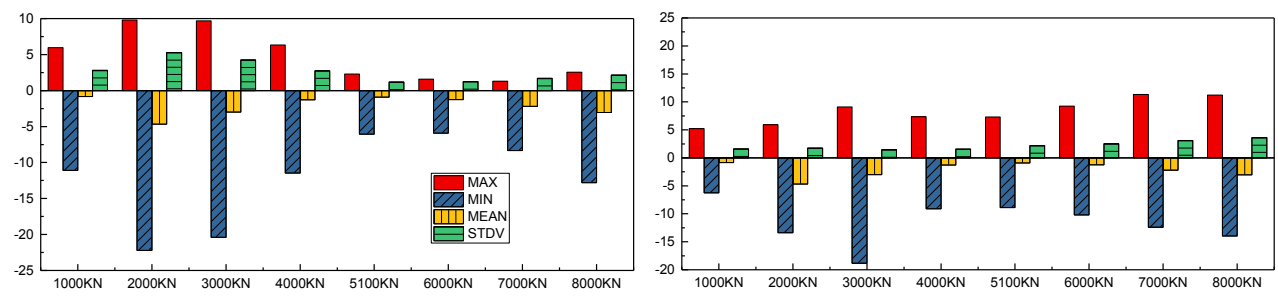

Fig. 11. Stats of relatvie motions of $Z 0=7 \mathrm{~m}$. (left : low frequency, right : wave frequency). 

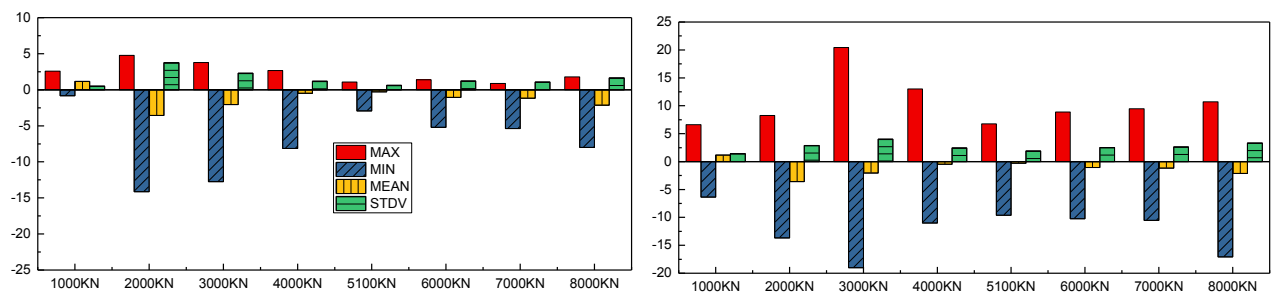

Fig. 12. Stats of relatvie motions of $Z_{0}=8 \mathrm{~m}$. (left : low frequency, right : wave frequency).
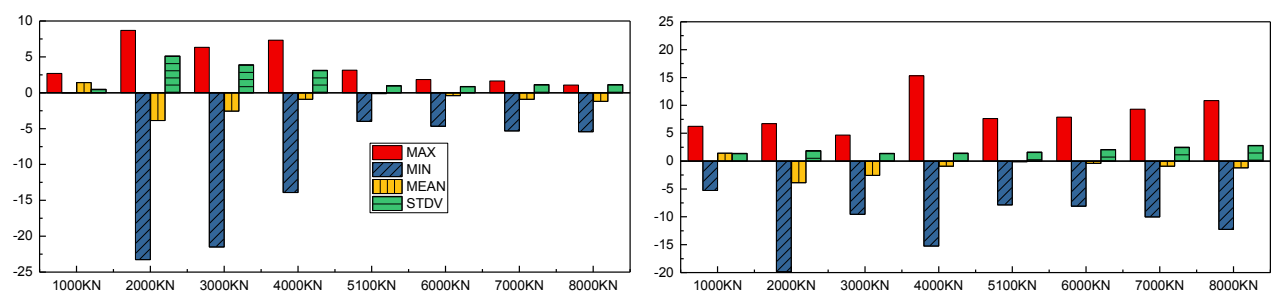

Fig. 13. Stats of relatvie motions of $Z_{0}=9 \mathrm{~m}$. (left : low frequency, right : wave frequency).

The estimation of the stroke range for each case is displayed in Fig.14 and values are provided in Table 4. Obviously, at lower pretension, all three nominal lengths will achieve better results. By increasing the pretension, larger relative motions are obtained. While at a certain range, like pretension equals to $5100 \mathrm{kN}$, for $\mathrm{Z}_{0}=8$ and $9 \mathrm{~m}$, acceptable results are obtained. Therefore, in this study, promising combinations among all the tested parameters are achieved. They are $Z_{0}=8 / 9 \mathrm{~m}$ under pretension $500 / 1000 \mathrm{kN}$ and $5100 \mathrm{kN}$.

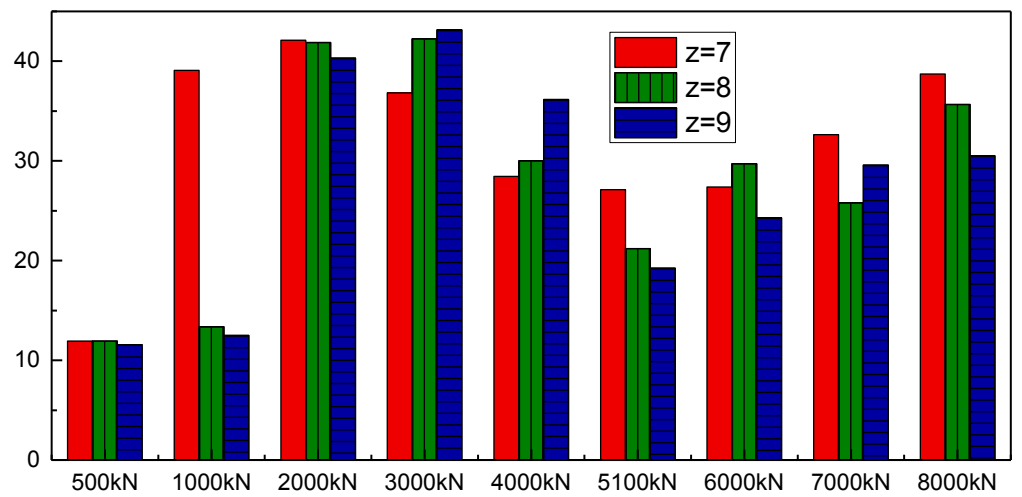

Fig. 14. Estimation of the stroke range of the coupling arm under different pretension.

Table 4 Data of the stroke range of the coupling arm.

\begin{tabular}{cccccccccc}
\hline$(\mathrm{m})$ & $500 \mathrm{kN}$ & $1000 \mathrm{kN}$ & $2000 \mathrm{kN}$ & $3000 \mathrm{kN}$ & $4000 \mathrm{kN}$ & $5100 \mathrm{kN}$ & $6000 \mathrm{kN}$ & $7000 \mathrm{kN}$ & $8000 \mathrm{kN}$ \\
\hline $\mathrm{Z}_{0}=7$ & 11.92 & 39.08 & 42.1 & 36.84 & 28.46 & 27.11 & 27.37 & 32.63 & 38.73 \\
$\mathrm{Z}_{0}=8$ & 11.94 & 13.35 & 41.87 & 42.24 & 30.01 & 21.22 & 29.72 & 25.78 & 35.66 \\
$\mathrm{Z}_{0}=9$ & 11.56 & 12.48 & 40.31 & 43.13 & 36.16 & 19.23 & 24.28 & 29.57 & 30.51 \\
\hline
\end{tabular}




\section{Conclusion}

The coupled TAD-TLP connected by an innovative rigid coupling arm is modelled by HARP with a user-defined external subroutine. A comprehensive parametric study is conducted to investigate the influence of the pretension and nominal lengths on the performance of the coupling arm, as well as the hydrodynamic performances. It is found that at low pretension, relative motion of the coupling arm at wave frequency dominates. As the pretension increases, the low frequency will play a more important role and lead to larger relative motions within a certain range. From this parametric study, promising combinations, $\mathrm{Z}_{0}=8 / 9 \mathrm{~m}$ under pretension $500 / 1000 \mathrm{kN}$ and $5100 \mathrm{kN}$ are achieved.

The authors thank the National Research Foundation, Keppel Corporation and National University of Singapore for supporting this work done in the Keppel-NUS Corporate Laboratory. The conclusions put forward reflect the views of the authors alone, and not necessarily those of the institutions within the Corporate Laboratory.

\section{References}

1. Y. Inoue, \& M. R. Islam, Comparative Study of Numerical Simulation And The Experimental Results For A Parallely Connected FPSO and LNG In Waves. In The Ninth International Offshore and Polar Engineering Conference. International Society of Offshore and Polar Engineers (1999)

2. M. C. Fang \& G. R. Chen, The Relative Motion and Wave Elevation between Two Floating Structures in Waves. In The Eleventh International Offshore and Polar Engineering Conference. International Society of Offshore and Polar Engineers. (2001)

3. B. Buchner, A. Van Dijk, \& J. De Wilde, Numerical multiple-body simulations of sideby-side mooring to an FPSO. In The Eleventh International Offshore and Polar Engineering Conference. International Society of Offshore and Polar Engineers. (2001)

4. Y. R. Choi, \& S. Y. Hong, An Analysis Of Hydrodynamic Interaction of Floating Multi-Body Using Higher-Order Boundary Element Method. In The Twelfth International Offshore and Polar Engineering Conference. International Society of Offshore and Polar Engineers. (2002)

5. J. Xia, \& R. Taghipour, Feasibility of TLP with Tender Assisted Drilling for Northwest Australian Waters-A Case Study. In Offshore Technology Conference. Offshore Technology Conference. (2012)

6. J. A. Pinkster \& G., Van Oortmerssen, Computation of the first- and second-order wave forces on oscillating bodies in regular waves. Proc. 2nd Int. Conf. Num. Ship Hydrodynamics, 136-156. (1977)

7. A. Jain, A. A. Hussain, \& A. A. Patent No. 9,272,755. Washington, DC: U.S. Patent and Trademark Office, Merchant, U.S. (2016)

8. C.K. Yang, A. Tahar, M.H. Kim, Linear and nonlinear approach of hydro-pneumatic tensioner modeling for spar global performance. In: Proceedings of Offshore Mechanics and Artic Engineering (OMAE07) \#29209, San Diego, California. (2007) 